\title{
Pulmonary magnetic resonance imaging in systemic sclerosis: a jump in the future to unravel inflammation in interstitial lung disease
}

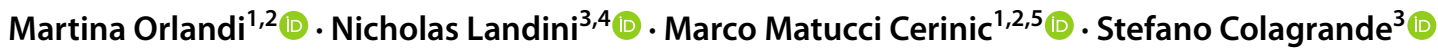

Received: 13 July 2021 / Revised: 20 July 2021 / Accepted: 22 July 2021 / Published online: 30 July 2021

(c) International League of Associations for Rheumatology (ILAR) 2021

Systemic sclerosis (SSc) is characterized by microvascular injury, inflammation and immune system activation, and an abnormal tissue deposition of extracellular matrix [1]. Inflammation and vascular injury, leading to vasculopathy, are the early cornerstones, thereafter, resulting in fibrosis of internal organs and their functional failure [2-4]. These events occur also in the lung, resulting in interstitial lung disease (ILD) [5, 6]. In SSc, ILD is a severe organ complication [7] and chest computed tomography (CT) is the most suitable, rapid, reliable technique for its evaluation and follow-up [8]. Moreover, the most recent CT scans can guarantee high spatial resolution also with low-dose acquisitions in a single breath-hold [9]. However, low-dose single CT examinations may suffer from a "cumulative dose", especially in the case of young patients, during the long-standing follow-up. It is well known that CT has some limitations,

Martina Orlandi

martina.orlandi@unifi.it

Nicholas Landini

nicholas.landini@hotmail.it

Marco Matucci Cerinic

marco.matuccicerinic@unifi.it

Stefano Colagrande

stefano.colagrande@unifi.it

1 Department of Clinical and Experimental Medicine, University of Florence, Florence, Italy

2 Department of Geriatric Medicine, Division of Rheumatology AOUC, Padiglione 28c Ponte Nuovo, piano 1. Via delle Oblate, 4, Firenze, Florence, Italy

3 Department of Experimental and Clinical Biomedical Sciences, and Radiodiagnostic Unit N. 2, University of Florence-Azienda Ospedaliero-Universitaria Careggi, Largo Brambilla 3, 50134 Florence, Italy

4 Department of Radiology, Ca' Foncello General Hospital, Piazzale Ospedale, 1, 31100 Treviso, Italy

5 Unit of Immunology, Rheumatology, Allergy and Rare Diseases (UnIRAR), IRCCS San Raffaele Hospital, Milan, Italy due to the risk of contrast agent administration and to the lack of real "functional" evidence, like ventilation and perfusion. In fact, dynamic or perfusional CT acquisition is possible but burdened by higher dose exposure like pneumo-CT and perfusional studies.

In order to decrease radiation burden during prolonged repetitive follow-up in young patients, lung ultrasound (LUS) was recently proposed both for screening and followup in ILD-SSc patients. LUS has shown high sensitivity for ILD detection by assessing the presence of B lines, vertically extending hyperechoic artifacts originating from the pleura (described as "comet tails"), that represents an indirect sign of ILD [10-12]. LUS has also been used to assess the severity of ILD by correlating the number of B lines with the extent of ILD on HRCT [11] and with the change in diffusing capacity for carbon monoxide [13]. Despite LUS being a non-invasive, low-cost, easily learned technique, the lack of methodological standardization, the incomplete field of view, and the low specificity limit its use in clinical practice. Moreover, LUS cannot detect ground glass opacities (GGO) and this is an important limit, in particular because GGO results as one of the earlier sign of ILD in SSc patients. According to the Fleischner Society glossary, GGO is defined as "hazy increased opacity, with preservation of bronchial and vascular margins" [14]. Usually, GGO is characterized by areas of hazy increased attenuation of the lung with preservation of bronchial and vascular margins and can represent either interstitial or alveolar processes [15]. On this background, it should be underlined that GGO represents an important limit at CT examination, as well. In fact, CT can detect very well GGO but cannot differentiate if it is due to an inflammatory or fibrotic condition.

Actually, from a histopathologic standpoint, GGO may be the result of partial filling of alveolar spaces or thickening of the alveolar walls/septal interstitium, or a combination of both. This pattern may be generated either by early inflammatory or late fibrotic changes of the lung parenchyma. For this reason, in the clinical setting, the 
interpretation of the GGO may be tricky, because it is not possible to definitively define GGO as mainly inflammatory or fibrotic. This fact may significantly limit the clinician in the choice of the therapeutic strategy (immunosuppressants vs antifibrotics).

Therefore, despite that CT is the golden standard for ILD evaluation, still today GGO remains a clinical challenge. On $\mathrm{CT}$, partially matching histopathologic features, GGO may be due to the reduction of air in the alveolar airspaces, partial filling of the alveolar airspaces, thickening of the parenchymal interstitium and alveolar walls, a relative increase in perfusion, or a combination of these factors. In order to fill up this gap, MRI may be seen as a powerful imaging tool, a radiation-free technique which may provide a discriminatory insight about the inflammatory or fibrotic nature of GGO.

Previously, MRI of the pulmonary parenchyma has been considered as a contradictory evaluation due to the extremely low proton density of lung tissue, to the magnetic susceptibility of sharp air/parenchyma interfaces and respiratory motion artifacts.

Recently, the new ultra-rapid MR sequences, such as the ultrashort echo-time (UTE), may overcome these limitations, counteracting lung parenchyma signal decay $\mathrm{T} 2$ star related [14-16]. Even if the geometric resolution of CT remains greatly superior to that of MRI, data from comparative studies have shown that, in ILD-GGO, UTE MRI provides comparable results to CT. Moreover, the speed of this kind of sequence, compared with the usual longer MRI acquisitions, may increase patient's tolerability to the exam and reduced costs. UTE are acquired during free breathing conditions with fully automatic respiratory synchronization and isotropic voxels, in a scan time of about $8 \mathrm{~min}$, and allow for both GGO detection and extent evaluation in every plan, by the multiplanar reconstructions permitted by isotropic voxels, with low incidence of major artifacts.

Usually, free breathing scans are obtained at functional residual volume, and therefore it is easily understandable that high-resolution breath-hold scans that match the inspiratory level and resolution of CT are highly desired. These techniques have been also combined to fast acquisition strategies, such as Compressed-Sensing (CS) VolumetricInterpolated-Breath-hold Examination (VIBE), to obtain breath-hold imaging. CS acquisitions allow the collection of the entire lung volume in breath-hold acquisitions of about $10 \mathrm{~s}$. However, breath-hold scans duration may be increased up to $20 \mathrm{~s}$, to improve both robustness towards respiratory motion and temporal resolution. Though, it was experienced that $20 \mathrm{~s}$ of apnea may be poorly tolerated by the great part of the non-early stage SSc-ILD patients and are scarcely reliable for ILD evaluation. At the moment, research groups are testing UTE and CS-VIBE sequences in SSc-ILD and it seems the better results are obtainable by free-breath UTE acquisitions $[17,18]$.
One of the first papers to investigate the role of MRI in assessing ILD on SSc patients was written by Pinal-Fernandez et al. [19] used a 2D BH half-Fourier single-shot TSE sequence (HASTE) in eighteen SSc patients. This sequence showed high sensitivity to detect SSc-ILD and was correlated with lung functional parameters (forced vital capacity, and diffusing capacity of the lung for carbon monoxide) and also with HRCT. However, MRI examination's extent were consistently lower than HRCT and, thus, not directly comparable. These data confirmed those by Ohno et al. [20] who showed that mean $\mathrm{T} 2$ values are different when comparing connective tissue disease ILD and healthy subjects, with significant correlation with diffusing capacity of the lung for carbon monoxide and severity of ILD on HRCT. Later, also Gargani et al. [21] confirmed the potential role of lung MRI in the detection of ILD in SSc patients, analyzing STIR and T1 mapping sequences (before and after gadolinium injection) in $30 \mathrm{SSc}$ patients. They also evaluated MR signal intensity in STIR and T1-weighted sequences in anatomical sections corresponding to normal lung parenchyma and ILD at CT scans reporting a significant difference between normal and pathological ILD lung areas. Moreover, STIR values are also able to predict worsening of ILD over time, independently of HRCT appearance. Miller et al. [22] tried to differentiate GGO with active pulmonary disease from non-active pulmonary disease, with only fibrotic lesions in 24 SSc patients, indeed they documented only a high agreement between MRI and CT.

Today, the main clinical question is whether MRI may differentiate inflammation from fibrosis in the context of GGO. Theoretically, at MRI, a predominantly inflammatory GGO rich in fluids (exudate) and vessels might give a different imaging than fibrosis, which is characterized by tissue rich in collagen and poor in fluid and vessels. In fact, MRI is very sensitive either in assessing the aqueous content or in recognizing fibrosis. For example, diffusion-weighted images (DWI) may give information on fluid presence and its microscopic motion (influenced by surrounding tissue components), as well as on microcirculation [23]. As far as we know, no data in the literature address this problem even if several attempts (also by us - Figs. 1 and 2) are ongoing.

The administration of the contrast agent could enhance GGO assessment as well as the presence of vessels, but several problems await a solution together with the resting state (RS), the low signal-to-noise ratio (SNR), the artifacts inherent in the presence of air, and the neighboring heartbeat. In the clinical setting, MRI might also detect not only an inflammatory GGO but more likely a "mixed" GGO, where inflammation and fibrosis overlap in different percentage according to the disease activity and evolution. The problem of identifying patients with a progressing phenotype is today of paramount importance in SSc-ILD [5]. Before a largescale prospective study is designed, a retrospective study 
Fig. 1 Early inflammatory interstitial lung disease in SSc. CT (A) and MR (B, UTE acquisition; $\mathbf{C}$, DwI acquisition; D, ADC map) studies. Early inflammatory involvement (black arrows and oval shaped area) with tiny GGO aspects, well demonstrated by CT (A). It can be seen that conspicuity of the involvement at UTE (B) acquisition is even better than that at CT. In the same area, DwI (C) shows medium intensity signal, which corresponds to an elevated value (around $2000 \cdot 10^{-6} \mathrm{~mm}^{2} / \mathrm{s}$ ) on the ADC map (D). This can agree with a high liquid presence in the flogistic lesions
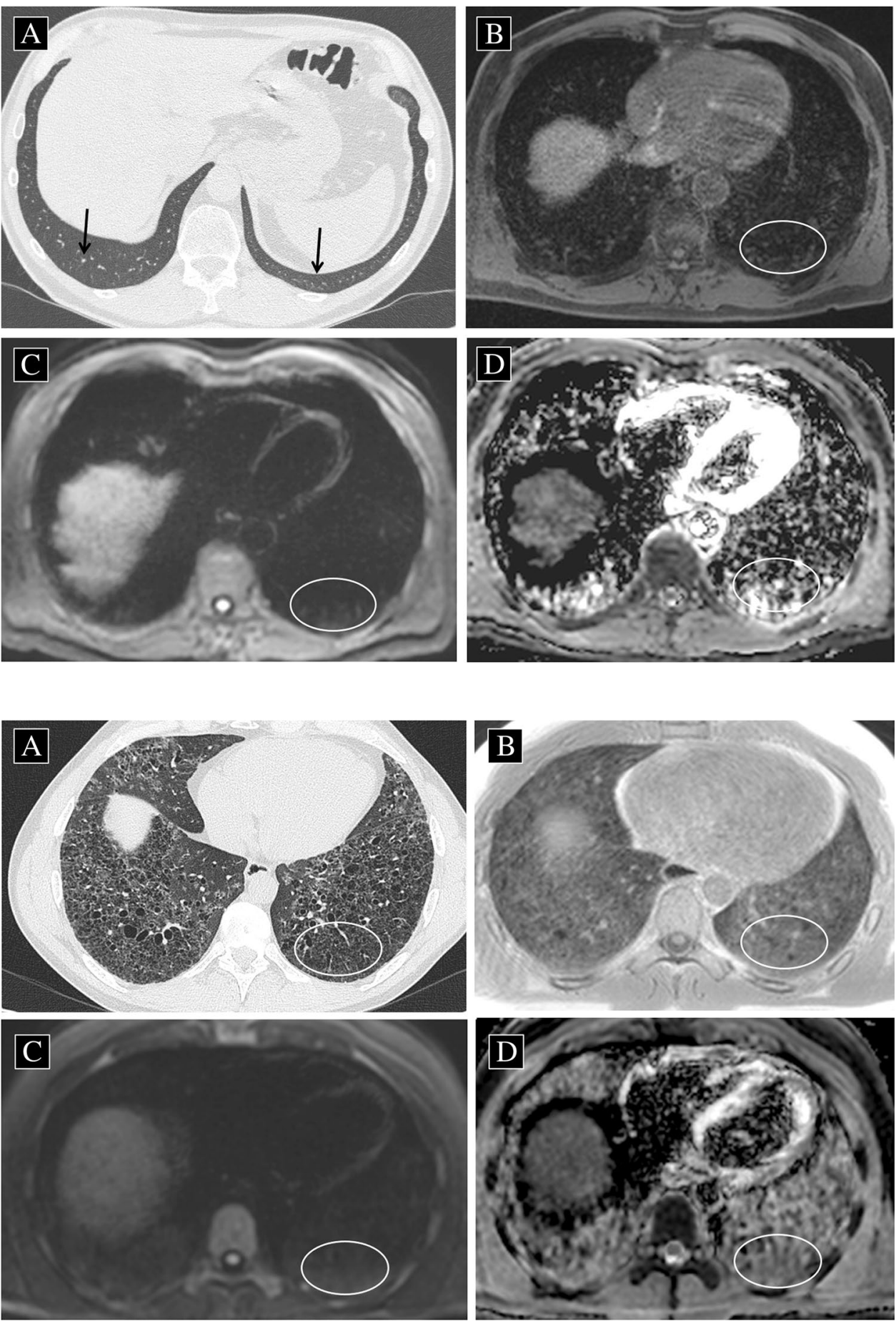

Fig. 2 Fibrotic interstitial lung disease in SSc. CT (A) and MR (B, UTE acquisition; C, DwI acquisition; D, ADC map) studies. Fibrotic involvement (oval shaped area) with some GGO aspects, demonstrated very well by $\mathrm{CT}(\mathbf{A})$ and quite well by UTE (B) acquisition. In the same area, DwI (C) shows medium intensity signal, which corresponds to a quite low value $\left(1200-1300 \cdot 10^{-6} \mathrm{~mm}^{2} / \mathrm{s}\right)$ on the ADC map (D). This can agree with a low liquid presence in the fibrotic tissue on cases defined as predominantly fibrotic or predominantly inflammatory is needed. The comparison with the results of the biopsy is still unlikely, due to its rare use.

In conclusion, while CT remains the gold standard for ILD diagnosis and assessment in the next future MRI may solve the problem of the interpretation of GGO, thus unraveling inflammation and allowing a targeted treatment and a follow-up with an x-ray sparing approach.
Authors' contributions $\mathrm{MO}, \mathrm{NL}, \mathrm{SC}$, and MMC conceived of the presented idea. All authors contributed to the final version of the manuscript.

Funding 'Not applicable'.

Data availability 'Not applicable'. 
Code availability 'Not applicable'.

\section{Declarations}

Disclosures None.

\section{References}

1. Orlandi M, Lepri G, Damiani A et al (2020) One year in review 2020: systemic sclerosis. Clin Exp Rheumatol 38(Suppl 125(3)):3-17

2. Trojanowska M (2010) Cellular and molecular aspects of vascular dysfunction in systemic sclerosis. Nat Rev Rheumatol 6:453-546

3. Matucci-Cerinic M, Kahaleh B, Wigley FM (2013) Review: evidence that systemic sclerosis is a vascular disease. Arthritis Rheum 65:1953-1962

4. Campbell PM, LeRoy EC (1975) Pathogenesis of systemic sclerosis: a vascular hypothesis. Semin Arthritis Rheum 4:351-368

5. Volkmann ER (2020) Natural history of systemic sclerosis-related interstitial lung disease: how to identify a progressive fibrosing phenotype. J Scleroderma Related Dis 5:31-40

6. Nihtyanova SI, Denton CP (2020) Pathogenesis of systemic sclerosis associated interstitial lung disease. J Scleroderma Related Dis 5(2_suppl):6-16

7. Volkmann ER, Fischer A (2021) Update on morbidity and mortality in systemic sclerosis-related interstitial lung disease. J Scleroderma Related Dis 6(1):11-20

8. Clukers J, Lanclus M, Belmans D et al (2021) Interstitial lung disease in systemic sclerosis quantification of disease classification and progression with high-resolution computed tomography: an observational study. J Scleroderma Relat Dis 6(2):154-164

9. Kuo W, Kemner-van de Corput MPC, Perez-Rovira A et al (2016) Multicenter chest computed tomography standardisation in children and adolescents with cystic fibrosis: the way forward. Eur Respir J 47:1706-1717

10. Barskova T, Gargani L, Guiducci S et al (2013) Lung ultrasound for the screening of interstitial lung disease in very early systemic sclerosis. Ann Rheum Dis 72:390-395

11. Hassan RI, Lubertino LI, Barth MA et al (2019) Lung ultrasound as a screening method for interstitial lung disease in patients with systemic sclerosis. J Clin Rheumatol 25(7):304-307

12. Edis EC, Hatipoğlu ON, Pamuk ON et al (2016) Effectiveness of thoracic ultrasonography in the evaluation of the severity of pulmonary involvement in patients with systemic sclerosis. Arch Rheumatol 31(4):364-370

13. Gasperini ML, Gigante A, Iacolare A et al (2020) The predictive role of lung ultrasound in progression of sclero-derma interstitial lung disease. Clin Rheumatol 39(1):119-123

14. Hansell DM, Bankier AA, MacMahon H et al (2008) Fleischner Society: glossary of terms for thoracic imaging. Radiology 246:697-722

15. Battista G, Sassi C, Zompatori M et al (2003) Ground-glass opacity: interpretation of high resolution CT findings. Radiol Med 106:425-442

16. Miller GW, Mugler JP, Sá RC et al (2014) Advances in functional and structural imaging of the human lung using proton MRI. NMR Biomed 27:1542-1556

17. Dournes G, Menut F, Macey J et al (2016) Lung morphology assessment of cystic fibrosis using MRI with ultra-short echo time at submillimeter spatial resolution. Eur Radiol 26:3811-3820

18. Ohno Y, Koyama H, Yoshikawa T et al (2016) Pulmonary highresolution ultrashort TE MR imaging: comparison with thinsection standard- and low-dose computed tomography for the assessment of pulmonary parenchyma diseases. J Magn Reson Imaging 43:512-532

19. Pinal-Fernandez I, Pineda-Sanchez V, Pallisa-Nuñez E et al (2016) Fast 1.5 T chest MRI for the assessment of interstitial lung disease extent secondary to systemic sclerosis. Clin Rheumatol 35:2339-2345

20. Ohno Y, Nishio M, Koyama H et al (2013) Pulmonary MR imaging with ultra-short TEs: utility for disease severity assessment of connective tissue disease patients. Eur J Radiol 82(8):1359-1365

21. Gargani L, Bruni C, De Marchi D et al (2021) Lung magnetic resonance imaging in systemic sclerosis: a new promising approach to evaluate pulmonary involvement and progression. Clin Rheumatol 40(5):1903-1912

22. Muller CS, Warszawiak D, Paiva EDS et al (2017) Pulmonary magnetic resonance imaging is similar to chest tomography in detecting inflammation in patients with systemic sclerosis. Rev Bras Reumatol Engl Ed 57(5):419-424

23. Colagrande S, Belli G, Politi LS et al (2008) The influence of diffusion- and relaxation-related factors on signal intensity: an introductive guide to magnetic resonance diffusion-weighted imaging studies. J Comput Assist Tomogr 32(3):463-474

Publisher's note Springer Nature remains neutral with regard to jurisdictional claims in published maps and institutional affiliations. 